\title{
Reconstructing the Topology of Protein Complexes
}

\author{
Allister Bernard ${ }^{\star}$, David S. Vaughn ${ }^{\star}$, and Alexander J. Hartemink \\ Department of Computer Science, Duke University, Durham, NC 27708-0129 \\ \{allister, dsv, amink\}@cs.duke.edu
}

\begin{abstract}
Recent advances in high-throughput experimental techniques have enabled the production of a wealth of protein interaction data, rich in both quantity and variety. While the sheer quantity and variety of data present special difficulties for modeling, they also present unique opportunities for gaining insight into protein behavior by leveraging multiple perspectives. Recent work on the modularity of protein interactions has revealed that reasoning about protein interactions at the level of domain interactions can be quite useful. We present PROCTOR, a learning algorithm for reconstructing the internal topology of protein complexes by reasoning at the domain level about both direct protein interaction data (Y2H) and protein co-complex data (AP-MS). While other methods have attempted to use data from both these kinds of assays, they usually require that co-complex data be transformed into pairwise interaction data under a spoke or clique model, a transformation we do not require. We apply PROCTOR to data from eight highthroughput datasets, encompassing 5,925 proteins, essentially all of the yeast proteome. First we show that PROCTOR outperforms other algorithms for predicting domain-domain and protein-protein interactions from Y2H and AP-MS data. Then we show that our algorithm can reconstruct the internal topology of AP-MS purifications, revealing known complexes like Arp2/3 and RNA polymerase II, as well as suggesting new complexes along with their corresponding topologies.
\end{abstract}

\section{Introduction}

Protein complexes serve as cellular building blocks, signal transducers, and machines. Protein complexes are assembled and held together by the direct interactions of their constituent proteins with one another. Direct interactions between pairs of proteins occurs in a modular fashion when some domain of one protein comes into sufficiently close proximity with some domain of the other such that the domains mediate an interaction between the two proteins 1

The structure of a macromolecular protein complex can be characterized at increasing levels of refinement: 1) identify its constituent proteins, 2) reveal its topology in terms of which proteins are directly interacting with which others, 3) determine the domain-domain interactions (DDIs) that mediate the direct protein-protein interactions (PPIs), and 4) specify the complete 3D atomic structure. While the fourth has become

\footnotetext{
* These authors contributed equally to this work.

${ }^{1}$ This interaction can be mediated by domains, motifs, or other features of the surface of a protein. Although not entirely precise, to simplify the presentation we make no distinction between any of these terms, but rather use the term 'domain' to refer to them interchangeably.
} 
easier for individual proteins, in the case of protein complexes, technical difficulties have posed significant obstacles to accomplishing this task [1]. Approaches are therefore needed at all these levels to help elucidate the architecture of complexes and how their individual subunits interact with each other [2].

\subsection{Related Work}

Recently, high-throughput PPI assays like yeast two-hybrid (Y2H) or affinity purification-mass spectrometry (AP-MS) have been conducted to partially bridge this gap. $\mathrm{Y} 2 \mathrm{H}$ assays use a gene reporter construct to screen for direct interactions between pairs of proteins. AP-MS assays, on the other hand, provide a list of 'prey' proteins that are identified by mass-spectrometry to have been co-complexed with a given 'bait' protein during an affinity purification process.

$\mathrm{Y} 2 \mathrm{H}$ and AP-MS assays have critically different semantics in terms of the kind of evidence they provide about PPIs. In contrast to Y2H, AP-MS assays do not provide direct evidence of PPIs but only indirect evidence. This means that correctly reasoning about both kinds of data together is challenging. Most methods tend to use either one kind of data or the other, in each case possibly supplementing the data with information from small scale experiments made available by databases like MIPS [27], DIP [38], and BIND [3]. Almost all existing methods for reasoning about both kinds of data do so by simply transforming the list of co-complexed proteins in an AP-MS purification into a list of direct protein interactions using either a 'spoke' or 'clique' model.

In the spoke model, a PPI is assumed to occur only between the bait and each prey protein. In the clique model, a PPI is assumed to occur between each pair of proteins (bait or prey). In general, a spoke model results in a lower false positive rate for PPIs within a complex at the expense of a higher false negative rate. On the other hand, a clique model results in a zero false negative rate for PPIs at the expense of a high false positive rate. If, however, the topology of PPIs explaining the AP-MS purification could be ascertained more accurately, then more accurate DDI estimates could be obtained as well. To the best of our knowledge, our approach is the first attempt to integrate AP$\mathrm{MS}$ and $\mathrm{Y} 2 \mathrm{H}$ assays by taking into account the different possible topologies of protein complexes that might explain a given AP-MS purification.

While Y2H and AP-MS assays differ importantly in terms of their semantics, they are similar in one distressing respect. For a range of published Y2H datasets and AP-MS datasets transformed by a spoke or clique model, error rates have been estimated to be significant: many true PPIs are not reported (70-98\%) and many reported PPIs are not true (46-90\%). Numerous computational methods have been developed to improve our knowledge of PPIs. Such methods have used a variety of techniques such as Bayesian networks [22], probabilistic decision trees [39], or kernel methods [5, 26, 18]. All of these improve prediction by incorporating multiple sources of additional information such as gene expression, Gene Ontology (GO) annotations, or interacting homologues of other species. Recent work has also examined the orthogonal problem of predicting co-complexed proteins from noisy AP-MS data [31, 8].

An alternative approach models the interactions between proteins' constituent domains to improve predictions. Here, the intuition is to use overrepresentation of certain DDIs among observed PPIs to more accurately predict both DDIs and PPIs. Such an 
approach was first outlined by Sprinzak and Margalit [32]. Deng and colleagues presented a probabilistic formulation in which an iterative EM algorithm was used to (locally) maximize a likelihood function [10]. Wang and colleagues extended this maximum likelihood formulation by incorporating the notion of 'active' interacting motifs [36]. Other approaches have explored Markov chain Monte Carlo methods [19] as well as the use of protein structure information [29, 30].

Here, we introduce a new statistical learning approach, PROCTOR (PROtein Complex TOpology Reconstruction), to elucidate the architecture of protein complexes, enabling effective use of the information available from both Y2H and AP-MS assays for reasoning about PPIs and DDIs. Our method provides a framework for determining the underlying topology of a protein complex and understanding how constituent proteins interact with each other to form a complex. Using available AP-MS datasets, including two recently published landmark datasets [16, 24], we determine topologies for macromolecular complexes containing essentially all of the yeast proteome. In addition, PROCTOR serves as a tool for predicting the DDIs that mediate PPIs. It accurately estimates DDIs across almost the entire yeast proteome and is efficient, displaying rapid convergence properties.

\section{Motivation and Intuition}

Our current knowledge of PPIs and DDIs is both very noisy and very incomplete, which means that current estimates of the probability of a given PPI or DDI can often be wildly inaccurate. As explained earlier, approaches have been developed to increase the accuracy of our estimates by modeling interactions between a proteins' constituent domains. In most of these cases, the data available for estimation comes from experimental assays that provide direct evidence of PPIs, such as $\mathrm{Y} 2 \mathrm{H}$ assays.

In contrast, AP-MS assays do not provide direct evidence of PPIs. First, each prey protein appearing in a given AP-MS purification is either a true positive or a false positive in terms of whether it actually co-complexes with the bait protein in vivo. In addition, although each true positive prey must somehow co-complex with the bait, 1) not all true positive preys must co-complex with one another (the bait may participate in multiple complexes), and 2) not all true positive preys must interact directly with the bait (many interactions may be indirect, via other intermediating preys).

Of course, we do not know which preys are true positives; nor do we know which true positive preys interact directly with the bait; nor do we know, in the case of true positive preys that interact only indirectly with the bait, which other preys intervene. Another way of saying this is that although many possible 'explanations' of an AP-MS purification are possible (as shown in Figure 1), we are not sure which explanation is true. If we knew which explanation was true, we could use this information across many AP-MS purifications to robustly estimate both the DDIs that mediate all the observed PPIs, as well as the internal topology of protein complexes in vivo.

One way to approach this problem would be to assume in advance which one particular explanation is to be taken as true: many methods do this when they explain the results of an AP-MS purification by stipulating the existence of direct PPIs among the bait and preys via either a spoke or a clique model. In addition to the fact that these 

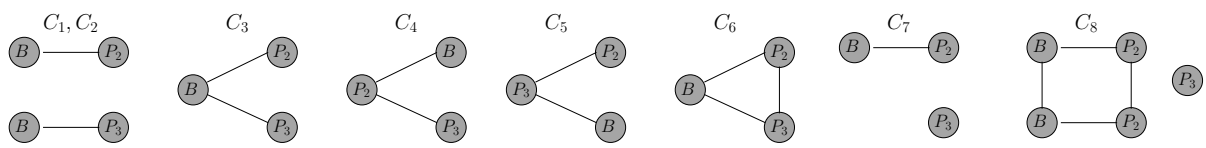

Fig. 1. Topologies of possible protein complexes when bait $B$ is purified along with two other proteins $P_{2}$ and $P_{3}$. Edges indicate PPIs. A spoke model assumes $C_{3}$, whereas a clique model assumes $C_{6}$. In $C_{7}$ and $C_{8}, P_{3}$ is a false positive. If multiple copies of proteins are permitted, many additional topologies are possible (e.g., $C_{8}$ ). In the absence of stoichiometric information, it is impossible to distinguish the two-complex case $C_{1}, C_{2}$ from the one-complex case $C_{3}$.

methods lead to poor estimates of the DDIs (because the stipulated PPIs are incorrect), such methods are incapable of estimating the topology of protein complexes because they assume the topology in advance.

A more sensible way to approach this problem is to estimate the probability of each possible explanation of the data. If this is done, a model selection perspective can be employed to choose the most likely explanation of the data, or alternatively, a more Bayesian model averaging perspective can be employed to marginalize over possible explanations of the data.

Here, we demonstrate how to compute the probability of each possible explanation of an AP-MS purification. We adopt a model averaging perspective to marginalize over possible explanations of the data in computing estimates for PPIs, and for the DDIs that mediate them. We also use this framework to reveal a model-averaged view of the internal topology of the protein complexes that give rise to the lists of proteins that appear in AP-MS purifications. In what follows, we first formulate the estimation problem for PPIs, DDIs, and unknown internal topologies, and then present a sampling algorithm for approximating the solution.

\section{Mathematical Formulation}

Let $\theta_{m n}$ be the probability of domains $m$ and $n$ interacting and let $\Theta=\left\{\theta_{m n}\right\}$ represent the set of all DDI probabilities. Likewise, let $w^{i j}$ denote the probability of proteins $i$ and $j$ interacting. Since we assume that a direct interaction between two proteins $i$ and $j$ is mediated by at least one interaction between some domain of $i$ and some domain of $j$, we can use a 'noisy-or' formulation to write:

$$
w^{i j}=1-\prod_{m, n}\left(1-\theta_{m n}\right)
$$

where $m$ and $n$ index over the constituent domains of proteins $i$ and $j$, respectively.

Define the false positive rate $\phi_{\mathrm{p}}$ as the probability of observing a prey protein in an AP-MS purification even though that prey does not actually co-complex with the bait in vivo; define the false negative rate $\phi_{\mathrm{n}}$ conversely: the probability of not observing a prey protein that actually co-complexes with the bait in vivo 2 We allow different

\footnotetext{
${ }^{2}$ Of course, complexes are dynamic and might exist only under certain conditions, so the concept 'actually co-complexes with' is ambiguous, but this subtlety is generally ignored in the literature—nor is the data available for addressing it—so we proceed to ignore it as well.
} 
AP-MS datasets to have different error rates, but assume within a single dataset that the false positive rate $\phi_{\mathrm{p}}$ is the same for all purifications, and also that the false negative rate $\phi_{\mathrm{n}}$ is the same for all purifications.

\subsection{Model for an AP-MS Purification Observation}

Denote the set of observed proteins in a single AP-MS purification as $\mathcal{O}$ (i.e., the bait protein plus all prey proteins). Consider the simplest (non-trivial) case, wherein bait $i$ is purified with only a single prey $j$. In this case, only two explanations are possibleeither the two proteins interact via one of their domains to form a two-protein complex or the proteins do not interact and the observed prey is a false positive. Given the DDI values $\Theta$ and the appropriate error rates, the complete probability of the observation is:

$$
\operatorname{Pr}\left(\mathcal{O} \mid \Theta, \phi_{\mathrm{n}}, \phi_{\mathrm{p}}\right)=\left(1-\phi_{\mathrm{n}}\right) w^{i j}+\phi_{\mathrm{p}}\left(1-w^{i j}\right)
$$

Now consider the case when an AP-MS purification contains more than two proteins. We first make the simplifying assumption that any complex topology for an AP-MS purification can only be a tree $c$ that spans over the complete graph induced over a subset $\mathcal{I}_{c}$ of the proteins $\mathcal{O}$. We require that the bait protein always be in $\mathcal{I}_{c}$. The remaining proteins $\mathcal{O}-\mathcal{I}_{c}$ are then treated as false positives of the AP-MS purification. The set of proteins $\mathcal{I}_{c}$ represent the true positives of the AP-MS purification and the edges $\mathcal{E}_{\mathcal{I}_{c}}$ that span over these true positives define an underlying topology for the complex(es) represented by this purification. We permit self-edges to be considered for proteins in $\mathcal{I}_{c}$ (true positives). For those concerned that self-edges violate the definition of a tree simply imagine that every protein is duplicated and a self-edge connects a protein $i$ to its duplicate protein. We call the tree $c$, consisting of interaction edges $\mathcal{E}_{\mathcal{I}_{c}}$ that span over $\mathcal{I}_{c}$ and the remaining false positive proteins $\mathcal{O}-\mathcal{I}_{c}$, a 'complex topology tree'. Biologically, such a tree represents the underlying interaction backbone for the set of complexes represented by this AP-MS purification. Note that throughout we use the terminology 'complex topology tree' but strictly speaking this represents the topology of multiple complexes represented by this purification. The probability of any complex topology tree $c$ is given by:

$$
\operatorname{Pr}(c)=\left[\left(1-\phi_{\mathrm{n}}\right)^{\left|\mathcal{I}_{c}\right|-1}\left(\phi_{\mathrm{p}}\right)^{\left|\mathcal{O}-\mathcal{I}_{c}\right|}\right]\left[\prod_{e^{i j \in \mathcal{E}_{\mathcal{I}_{c}}}} w^{i j}\right]\left[\prod_{e^{i j \notin \mathcal{E}_{\mathcal{I}_{c}}}}\left(1-w^{i j}\right)\right]
$$

This has three terms - the first term models the true and false positives represented by the tree, the second term models the interactions spanned by the tree, and the final term ensures that this is the probability of $c$ and only $c$ (and not graphs for which $c$ is a sub-graph). The probability of observing an AP-MS purification is the sum of the probabilities of all possible complex topology trees $c \in \mathcal{C}$ :

$$
\operatorname{Pr}\left(\mathcal{O} \mid \Theta, \phi_{\mathrm{n}}, \phi_{\mathrm{p}}\right)=\sum_{c \in \mathcal{C}} \operatorname{Pr}(c)
$$




\subsection{Incorporating Negative Information into the Observation Model}

The model for observing an AP-MS purification in (3) ignores the possibility of false negative complexes or false negative errors due to mass spectrometry. It also fails to recognize that proteins which do not participate in a complex with a bait provide negative evidence for DDIs. Let $\overline{\mathcal{O}}$ be the set of proteins that are neither the bait nor observed as prey proteins in some purification. Modeling false negative complexes would require an expression for the probability of $\overline{\mathcal{O}}$ analogous to that in (3), but this is not practical since the size of $\overline{\mathcal{O}}$ is many orders of magnitude larger than that of $\mathcal{O}$. Instead, we only incorporate negative evidence for pairs of proteins in the set $\mathcal{O} \times \overline{\mathcal{O}}$. This results in the following negative observation model:

$$
\operatorname{Pr}\left(\overline{\mathcal{O}} \mid \Theta, \phi_{\mathrm{n}}, \phi_{\mathrm{p}}\right)=\prod_{i \in \overline{\mathcal{O}}}\left[\left(1-\phi_{\mathrm{p}}\right) \prod_{j \in \mathcal{O}}\left(1-w^{i j}\right)+\phi_{\mathrm{n}} \sum_{j \in \mathcal{O}} w^{i j} \prod_{\substack{k \in \mathcal{O} \\ k \neq j}}\left(1-w^{i k}\right)\right]
$$

For each protein $i \in \overline{\mathcal{O}}$, the first term in the product models the possibility of $i$ being a true negative (and thus not interacting with any of the proteins in $\mathcal{O}$ ), while the second term models the possibility of $i$ being a false negative (in this case, we make a further simplification by assuming $i$ interacts with only one protein $j \in \mathcal{O}$ ).

\section{Joint Learning Using Proteomic Data}

In the preceding section, we developed a model for explaining an AP-MS purification. We note this is easily extended to incorporate data from $\mathrm{Y} 2 \mathrm{H}$ assays: we simply treat every observed $\mathrm{Y} 2 \mathrm{H}$ interaction as a two-protein complex whose probability of interaction is thus given by (1). For $\mathrm{Y} 2 \mathrm{H}$ datasets, we do not need to worry about incorporating negative evidence as every unobserved interaction $\overline{\mathcal{O}}$ is treated as a negative.

\subsection{Joint Model for Y2H and AP-MS Data}

Suppose we have $K \mathrm{Y} 2 \mathrm{H}$ datasets $Y_{k}$ and $L$ AP-MS datasets $A_{l}$. Let us denote all the Y2H datasets by $\mathcal{Y}=\left\{Y_{k} \cdot \mathcal{O}, Y_{k} . \mathcal{O}, Y_{k} . \phi_{\mathrm{p}}, Y_{k} . \phi_{\mathrm{n}}\right\}_{k=1}^{K}$ and all the AP-MS datasets by $\mathcal{A}=\left\{\left\{A_{l} \cdot \mathcal{O}, A_{l} \cdot \overline{\mathcal{O}}\right\}, A_{l} . \phi_{\mathrm{p}}, A_{l} \cdot \phi_{\mathrm{n}}\right\}_{l=1}^{L}$. In the definition of $\mathcal{A}$, we have used set notation (curly brackets) around the first two elements to remind the reader that each AP-MS dataset consists of a (large) set of purifications. We can now construct a full joint probability model of all these assays as shown:

$$
\begin{aligned}
& \operatorname{Pr}(\mathcal{Y}, \mathcal{A} \mid \Theta, \Phi)=\prod_{k=1}^{K}\left(\prod_{\left.\operatorname{Pr}\left(Y_{k} \cdot \mathcal{O} \mid \Theta, \Phi\right) \prod\left(1-\operatorname{Pr}\left(Y_{k} \cdot \mathcal{O} \mid \Theta, \Phi\right)\right)\right)}\right. \\
& \times \prod_{l=1}^{L}\left(\prod \operatorname{Pr}\left(A_{l} \cdot \mathcal{O} \mid \Theta, \Phi\right) \operatorname{Pr}\left(A_{l} \cdot \overline{\mathcal{O}} \mid \Theta, \Phi\right)\right)
\end{aligned}
$$

The first term represents the $\mathrm{Y} 2 \mathrm{H}$ model and is fully specified by (1). Here, the inner product terms are over all PPIs observed and unobserved in the $\mathrm{Y} 2 \mathrm{H}$ dataset $Y_{k}$, respectively. The second term represents the AP-MS model and is fully specified by (3) 
and (4). Here, the inner product is over all the purifications of the AP-MS dataset $A_{l}$. We point out that in the absence of AP-MS information, this model is equivalent to a previously published method [10]. The assumption of independence is strictly not correct but has been introduced to avoid the complicated dependency structure arising with AP-MS data. The independence assumption can be relaxed for $\mathrm{Y} 2 \mathrm{H}$ data and leads to a more efficient algorithm. For lack of space, to simplify presentation, and since we observed only a marginal improvement in results, we present these ideas in the Supplementary Material.

\subsection{Inference Using a Monte Carlo EM Algorithm}

We generalize the EM algorithm of [10] to incorporate our AP-MS observation model. As in [10], we assume the error rates $\Phi$ are given, so we only need to estimate the DDI probabilities $\Theta$. This is not a serious problem in practice, as estimates of error rates for a number of $\mathrm{Y} 2 \mathrm{H}$ and AP-MS datasets have been published [13, 35, 11, 17].

Exact computation of (3) requires the enumeration of all possible spanning trees in a complete graph which is $O\left(|\mathcal{O}|^{|\mathcal{O}|-2}\right)[25]$. This is clearly not tractable. Instead, we approximate (3) using a Monte Carlo approach by generating random trees from the uniform distribution and then calculate (3) using these trees. This can be implemented efficiently by performing a simple random walk over a complete graph with vertex set $\mathcal{O}$ [7, 37, 25]. Thus development of an efficient sampling algorithm requires that complex topologies only be trees. This is why we have made the simplifying assumption that the space of possible complex topologies can only be trees. Note that this assumption is not unduly restrictive as a complex topology that is a graph can be represented as a combination of a set of trees. To model false positives, we randomly select a set of vertices from $\mathcal{O}$ as false positives and then use the simple random walk on the remaining set of vertices. Care has to be taken that the selected set of trees are unique. This can be done efficiently by maintaining a hashtable indexed by a hash function dependent on the structure of the tree. The number of trees (samples) generated for an AP-MS purification is initially selected as a function of $A_{l} . \mathcal{O}$ to reflect the fact that the number of possible trees is a function of the size of the purification. This number is then uniformly increased at each iteration of the EM algorithm to increase the accuracy of the approximation over time.

We now outline the extensions needed to the EM algorithm of [10]. The E-step requires the calculation of the binomial sufficient statistics for the binary latent variables $D_{m n}^{i j}$ representing the presence of an interaction between domains $m$ and $n$ in proteins $i$ and $j$. For an AP-MS purification, we need to consider two cases.

a. When $(i, j) \in \mathcal{O} \times \mathcal{O}$, the E-step calculations are shown below, where $\mathbf{I}_{\left\{e^{i j} \in \mathcal{E}_{\mathcal{I}_{c}}\right\}}$ is an indicator variable which returns 1 if the edge $e^{i j}$ is in the edge set $\mathcal{E}_{\mathcal{I}_{c}}$ of tree $c$ and 0 otherwise.

$$
\begin{aligned}
E\left(D_{m n}^{i j} \mid \Theta^{(t-1)}, \Phi\right) & =\frac{\theta_{m n}^{(t-1)} \operatorname{Pr}\left(\mathcal{O} \mid D_{m n}^{i j}, \Theta^{(t-1)}, \Phi\right)}{\operatorname{Pr}\left(\mathcal{O} \mid \Theta^{(t-1)}, \Phi\right)} \\
\operatorname{Pr}\left(\mathcal{O} \mid D_{m n}^{i j}, \Theta^{(t-1)}, \Phi\right) & =\sum_{c \in \mathcal{C}} \frac{\operatorname{Pr}(c) \mathbf{I}_{\left\{e^{i j} \in \mathcal{E}_{\left.\mathcal{I}_{c}\right\}}\right.}}{w^{i j}}
\end{aligned}
$$


b. When $(i, j) \in \overline{\mathcal{O}} \times \mathcal{O}$, the E-step calculations are shown below.

$$
\begin{aligned}
E\left(D_{m n}^{i j} \mid \Theta^{(t-1)}, \Phi\right) & =\frac{\theta_{m n}^{(t-1)} \operatorname{Pr}\left(\overline{\mathcal{O}} \mid D_{m n}^{i j}, \Theta^{(t-1)}, \Phi\right)}{\operatorname{Pr}\left(\overline{\mathcal{O}} \mid \Theta^{(t-1)}, \Phi\right)} \\
\operatorname{Pr}\left(\overline{\mathcal{O}} \mid D_{m n}^{i j}, \Theta^{(t-1)}, \Phi\right) & =\phi_{\mathrm{n}} \prod_{\substack{k \in \mathcal{O} \\
k \neq j}}\left(1-w^{i k}\right)
\end{aligned}
$$

If the total number of possible interactions between domains $m$ and $n$ across all $\mathrm{Y} 2 \mathrm{H}$ and AP-MS datasets is $T_{m n}$, then the M-step involves a simple recursive formula for $\theta_{m n}$, where the summation is over all pairs of proteins $i$ and $j$ that contain domains $m$ and $n$, respectively.

$$
\theta_{m n}^{(t)}=\frac{1}{T_{m n}} \sum E\left(D_{m n}^{i j} \mid \Theta^{(t-1)}, \Phi\right)
$$

We now briefly examine the time and space requirements for each iteration of the EM algorithm. Let $P$ be the size of the proteome, $D$ be the total number of unique domains across all proteins, $S$ be the maximum number of samples (trees) generated for a particular EM iteration, $R$ be the total number of AP-MS purifications (this is the sum total of all purifications across the $L$ AP-MS datasets), $H$ be the size of the AP-MS purification with the maximum number of prey proteins, and $T$ be the maximum number of proteinprotein pairs with the same domain-domain combination (terms summed over in (8)). $\mathrm{Y} 2 \mathrm{H}$ and AP-MS datasets require $O(K P+R H)$ space (we assume that the number of observed protein-protein interactions in any $\mathrm{Y} 2 \mathrm{H}$ assay is orders of magnitude less than the total number of possible interactions). Each EM iteration involves maintaining $O\left(P^{2}\right)$ and $O\left(D^{2}\right)$ matrices for updating the sufficient statistics. Selecting a unique set of trees generated from the simple random walk requires $O(S H)$ space. Thus, the total space usage at each EM iteration is $O\left(K P+R H+P^{2}+D^{2}+S H\right)$. In practice, $H$ is usually orders of magnitude smaller than $P$ and $D$. For the rare AP-MS purifications with many prey proteins, we can set an upper bound on the value of $S H$ without adversely affecting the algorithm. Updating the sufficient statistics requires $O\left(P^{2}+D^{2} T\right)$ time. Generating a sample of random trees requires $O\left(S H^{2}\right)$ time (checking whether a tree is unique can be made $O(1)$ with a good hash function, the simple random walk requires $O(H \log H)$, and computing the cost of a tree requires $O\left(H^{2}\right)$ ). Thus, the time of each EM iteration is bounded by $O\left(P^{2}+D^{2} T+R H+S H^{2}\right)$. In practice, $T$ is not prohibitively large. Hence, both time and space requirements are dominated by the number of samples $S$ used in each iteration, the size of the proteome $P$, and the number of unique domains $D$.

\subsection{Recovering the Topology of a Protein Complex}

Our Monte Carlo EM algorithm provides estimates for the DDI probabilities $\Theta$. Given $\Theta$, we can compute the probabilities of different complex topologies. If we assume the topology is tree, we can determine the most probable such tree using standard minimum spanning tree (MST) algorithms over the complete graph with vertex set $\mathcal{O}$. The MST is essentially a maximum likelihood estimate of the complex topology, restricted to a tree. One might also wish to determine the most probable DDIs that mediate the PPIs in the 
graph. In this case, one could construct an MST in an annotated graph over the set of proteins $\mathcal{O}$ : instead of having a single edge between proteins $i$ and $j$, the annotated graph would have as many edges as the number of possible DDIs between $i$ and $j$. A natural extension to this would be to then determine a complex topology for the proteins in $\mathcal{O}$ such that edges selected for the spanning tree never re-use any of a protein's domains. We call this the Domain Re-use Constraint problem and have proved that it is NP-Hard (see Supplementary Material).

Using a maximum likelihood estimate as described above would prevent us from noticing if a different tree were nearly equally probable. We overcome this by using Bayesian model averaging instead, employing our sampling algorithm to generate a huge number of random trees and then computing marginal probability estimates for all the edges of the graph. Using this Bayesian model averaging approach, we are not restricted to complex topology trees but can determine complex topology graphs (i.e., with cycles). Note also that such topology graphs can represent the topology of more than one complex since proteins can participate in more than one complex.

\section{Results}

We applied our algorithm to publicly available high-throughput proteomic interaction data for $S$. cerevisiae. We obtained a total of 6,864 purifications from five different AP-MS datasets. Four datasets were tandem affinity purification (TAP) assays with 589 purifications [15], 294 purifications [23], 1,993 purifications [16], and 3,436 purifications [24], respectively. The fifth dataset was a high-throughput mass spectrometric protein complex identification (HMS-PCI) assay with 552 purifications [20]. The average size of the number of prey proteins across all 6,864 AP-MS purifications was 13, with a maximum size of 332 .

We also used two $\mathrm{Y} 2 \mathrm{H}$ datasets. The first included two $\mathrm{Y} 2 \mathrm{H}$ screens, one where all transformants were allowed to mate with each other $(6,000 \times 6,000)$ and another where all transformants were allowed to mate with only $192(6,000 \times 192)$ [34]. Ideally, we would treat these two screens as separate datasets, but the identities of the 192 transformants used in the second screen were not recorded (Peter Uetz, personal communication) so we pooled the two screens into one dataset containing 957 interactions. The second $\mathrm{Y} 2 \mathrm{H}$ dataset included one high-throughput screen $(6,000 \times 6,000)$ producing a total of 4,549 interactions, 841 of which were detected more than three times and labeled as 'core' interactions [21]. We treated the core and full sets as separate $\mathrm{Y} 2 \mathrm{H}$ datasets to reflect the difference in the confidence of their observations.

All the data taken together represent 5,925 yeast proteins, covering essentially the entire yeast proteome. We used protein-domain information from two different sourcesPfam [4] and InterPro [28] — and evaluated all our results with each of these sources independently. The average number of domains per protein was 2 for both Pfam and InterPro, with the maximum number of domains in a protein being 30 and 18, respectively.

For AP-MS assays, we used previously reported false positive and false negative error estimates [17]. To estimate error rates for direct protein interaction assays like Y2H (or AP-MS assays converted using spoke or clique models), we used a published 
method for simultaneously estimating the error rates of two datasets given a gold standard set of known interactions [11]. By comparing two independent datasets to a gold standard, the error rates are more trustworthy than those obtained by comparing each dataset to the gold standard by itself. To further increase our confidence in these values, we paired each dataset with several others, ran the method for each pair, and averaged all the results (see Supplementary Material for values).

Although the space and time complexity of our learning algorithm increases as the number of samples increase with each EM iteration, in practice the algorithm converged rapidly within 25-30 iterations, with each iteration taking an average of 30 minutes on a machine with 4GB RAM. Convergence was assessed as change in log likelihood being less than $0.01 \%$. We compared four algorithms: 1 ) a previously published algorithm [10] using $\mathrm{Y} 2 \mathrm{H}$ datasets only (called Y $2 \mathrm{H}-\mathrm{ONLY}$ ), 2) the same algorithm as 1 but including AP-MS data transformed using a spoke model (called SPOKE), 3) the same algorithm as 1 but including AP-MS data transformed using a clique model (called CLIQUE) and 4) our algorithm using separate observation models for Y2H and AP-MS datasets (called PROCTOR). We experimented with random initializations for $\theta_{m n}$ but consistently observed the best results by initializing $\theta_{m n}$ to a constant less than 0.01 . None of these algorithms displayed significant differences for small variations in the error rate estimates, as has been observed by others as well [10]. Also, the observed results were virtually indistinguishable whether we used protein-domain information from Pfam or Interpro, so we only show results using Pfam.

\subsection{Evaluation of Domain-Domain Interaction Predictions}

We used structural information from 3DID [33] and iPfam [14] to define positive DDIs for our evaluation set. As suggested previously [30], we pruned out those DDIs having no evidence in the training data because none of the algorithms will be able to do better than random for these, leaving us with a total of 1,501 positive DDIs for evaluation. We selected 40 times as many negative DDIs by randomly sampling from DDIs not in our positive set. Figure 2 shows the resulting precision-recall curves; we use precisionrecall curves instead of ROC curves since they are more informative when using highly skewed evaluation sets [9]. The SPOKE approach does a little better than the CLIQUE approach, while Y2H-ONLY fares the worst. This is presumably due to the fact that it has the least available training data. Overall, the area under the DDI precision-recall curve increases at least 0.36 with PROCTOR. On examining the curve for the CLIQUE approach more closely, we discovered its poor performance was partly due to its inability to predict self-interacting domains. We then modified CLIQUE to include self-interacting proteins (a protein interacts not only with all other proteins but also with itself). This modification improved the precision-recall curve for CLIQUE to slightly better than that of SPOKE and Y2H-ONLY (results not shown).

\subsection{Evaluation of Protein-Protein Interaction Predictions}

We obtained positive PPIs for our evaluation set from the small scale experiments contained in DIP [38]. We again pruned out PPIs having no evidence in the training data, leaving us with a total of 3,144 positive PPIs for evaluation. We selected 40 times as 


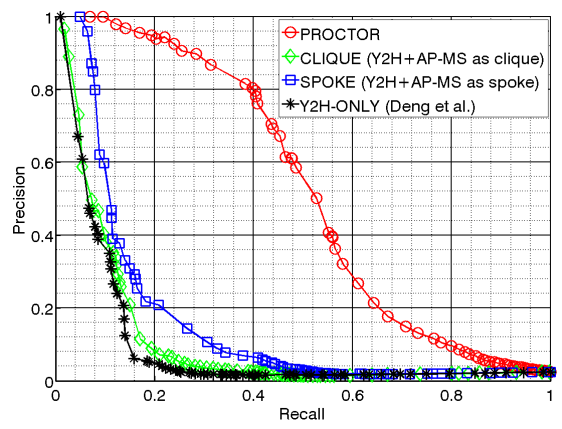

Fig. 2. DDI: precision vs. recall

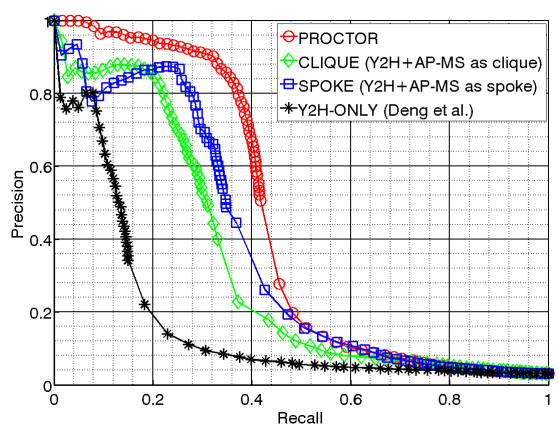

Fig. 3. PPI: precision vs. recall

many negative PPIs by randomly sampling from PPIs not in our positive set. As an alternative negative evaluation set, we also obtained the localization of 1,119 proteins [12] and randomly sampled from pairs of proteins with different localizations. These two negative evaluation sets showed no discernible difference (results not shown), so we only present results using the former (without localization). Somewhat surprisingly, the PPI precision-recall curves, shown in Figure 3, are not as dramatically different as the DDI precision-recall curves. Although PROCTOR still performs the best, the area under the PPI precision-recall curve increases only 0.08 compared to the next best algorithm (SPOKE). Upon close examination, we determined that this difference between DDI and PPI precision-recall curves is due to a combination of the inherently non-uniform contributions of different DDIs to the PPI network, the low prevalence of PPIs (the ratio of non-PPIs to PPIs in the proteome is estimated to be at least 1,200), and differences in the evaluation sets. Both SPOKE and CLIQUE predict many more DDIs than PROCTOR. However, these DDIs are distributed across a relatively small fraction of the PPIs. Both SPOKE and CLIQUE also predict many more PPIs than PROCTOR, but these additional PPIs are extremely small in number when compared to the total number of possible PPIs. As a result of all these factors, the improvement observed in Figure 3 is not as visually dramatic as that seen in Figure 2

\subsection{Prediction of Protein Complex Topologies}

Finally, we examine PROCTOR's ability to reconstruct the topology of protein complexes in an AP-MS purification. To construct a complex topology graph explaining a purification, we used our sampling algorithm to compute an average confidence for each edge in the graph. For two well-studied complexes, we compared the known crystal structure in PDB [6] with the topology predicted by PROCTOR. The first complex we studied is part of the core component of RNA polymerase II (PDBid: 1sfo) and the reconstructed complex topology is shown in Figure 4 Tfg1 was used as the bait in this purification and is involved in both transcription initiation and elongation of RNA polymerase II. Besides the five members of the core component (Rpb1, Rpb2, Rpb3, Rpb6, Rpb8), the Tfg1 purification pulled down two unrelated proteins, Hst4 and Msy1, both of which were correctly identified as false positives. The second complex we studied 

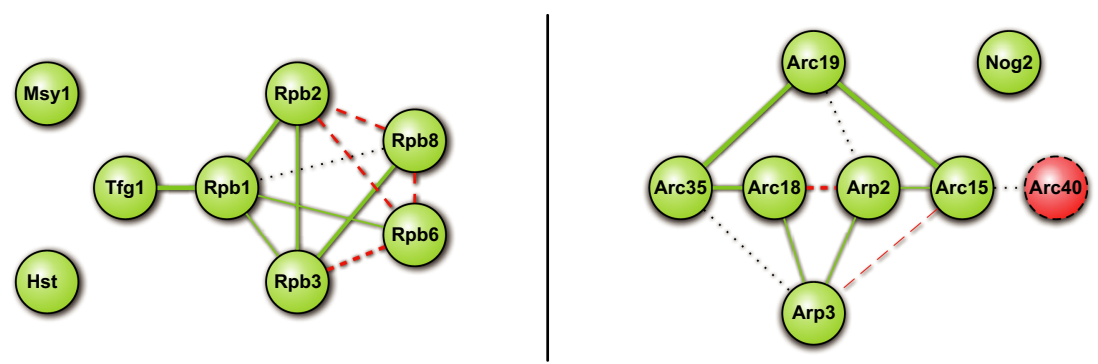

Fig. 4. Predicted complex topology of Arp2/3 complex and a portion of RNA polymerase II. The width of each edge reflects our relative confidence in the corresponding PPI. Dashed red nodes indicate nodes wrongly labeled as a false positive. Dashed red edges indicate PPIs wrongly labeled as interactions. Dotted black edges indicate PPIs wrongly labeled as non-interactions. All solid green nodes and solid green edges are correct.

is the Arp2/3 complex (PDBid: $1 \mathrm{k} 8 \mathrm{k}$ ) which is a highly conserved actin nucleation center consisting of seven proteins. We had AP-MS purifications with both Arp2 and Arp3 as bait. We reconstructed the same topology from both purifications, one of which is shown in Figure 4. Only Arc40 was not selected as part of the complex (incorrectly). We suspect this is because Arc40 appeared in the Arp2 purification, but not in the Arp3 purification. On the other hand, Nog2 was correctly identified as a false positive. Observe that the topology of neither complex can be accurately characterized by the spoke or clique models. In addition to reconstructing protein complex topologies, PROCTOR also provides an estimate of the DDIs that mediate the PPIs in these topologies, but this has been left out of the figure to reduce clutter.

\section{Discussion}

We demonstrate the accuracy of our learning algorithm PROCTOR for predicting both PPIs and the DDIs that mediate them, and more importantly, in reconstructing the topologies of protein complexes. These topologies are useful in providing a better understanding of the architecture of cellular protein complexes. This has been achieved by careful modeling of the different semantics of $\mathrm{Y} 2 \mathrm{H}$ assays (direct protein interaction evidence) and AP-MS assays (protein co-complex evidence). Our implementation of PROCTOR also permits variations within one kind of assay to be modeled with different error rates: e.g., TAP vs. HMS-PCI protocols used for AP-MS, or core vs. full for Y2H. Our results demonstrate that sizable improvements in accuracy can be obtained by careful modeling of the semantics of AP-MS data; if the distinct semantics of APMS data are ignored, algorithm performance degenerates rapidly. Our implementation of PROCTOR is also the first domain-domain model for protein-protein interactions to cover essentially all of the yeast proteome. Previously reported results [10, 36, 30] have less than half this coverage, and in no case cover more than $42 \%$ of the proteome.

The most important feature of PROCTOR is its ability to further our understanding of the architecture of cellular macromolecules through the reconstruction of complex 
topology graphs (along with the corresponding DDIs that mediate them). We plan to set up a database of PROCTOR's protein complex topology reconstructions as a useful tool for others in the community.

Currently, PROCTOR reports a topology for explaining each individual purification, ignoring potential overlap between purifications either due to repeated experiments or the use of different members of a complex as bait proteins. It would be useful to extend our algorithm to use such information for partitioning AP-MS purifications into their constituent complexes. We might be able to use ideas developed by others for predicting co-complex membership [31, 8] to assist in this. In addition, some of the available AP-MS datasets may soon be enhanced to include stoichiometric information for their purifications (Anne-Claude Gavin, personal communication). We would like to explore the use of such information in further elucidating the topology of protein complexes.

Acknowledgments. The authors would like to thank Ed Iversen and Kamesh Munagala for useful discussions and suggestions, and Carlo Tomasi for suggesting the name PROCTOR. The research presented here was supported by a National Science Foundation CAREER award and an Alfred P. Sloan Fellowship to A.J.H.

Supplementary Material can be found at http://www.cs.duke.edu/ amink/.

\section{References}

[1] P. Aloy, B. Bottcher, H. Ceulemans, C. Leutwein, C. Mellwig, S. Fischer, A.C. Gavin, P. Bork, G. Superti-Furga, L. Serrano, and R.B. Russell. Structure-based assembly of protein complexes in yeast. Nature, 303:2026-2029, 2004.

[2] P. Aloy and R.B. Russell. Structural systems biology: modeling protein interactions. Nature Reviews in Molecular Cell Biology, 7:188-197, 2006.

[3] G.D. Bader, I. Donaldson, C. Wolting, B.F. Ouellette, T. Pawson, and C.W. Hogue. BIND the biomolecular interaction network database. Nucleic Acids Research, 29:242-245, 2001.

[4] A. Bateman et al. The pfam protein families database. Nucleic Acids Research, 32: D138-D141, 2004.

[5] A. Ben-Hur and W.S. Noble. Kernel methods for predicting protein-protein interactions. In ISMB. ISCB, June 2005.

[6] H.M. Berman, J. Westbrook, Z. Feng, G. Gilliland, T.N. Bhat, H. Weissig, I.N. Shindyalov, and P.E. Bourne. The protein data bank. Nucleic Acids Research, 28: 235-242, 2000.

[7] A.Z. Broder. Generating random spanning trees. In Foundations of Computer Science, pages 442-447. IEEE, 1989.

[8] W. Chu, Z. Ghahramani, R. Krause, and D.L. Wild. Identifying protein complexes in highthroughput protein interaction screens using an infinite latent feature model. In PSB, pages 231-242, 2006.

[9] Jesse Davis and Mark Goadrich. The relationship between precision-recall and roc curves. In Proc. 23rd International Conference on Machine Learning, pages 233-240, 2006.

[10] M. Deng, S. Mehta, F. Sun, and T. Chen. Inferring domain-domain interactions from protein-protein interactions. In RECOMB '02: Proceedings of the sixth annual international conference on Computational biology, pages 117-126. ACM Press, 2002.

[11] P. D'haeseleer and G.M. Church. Estimating and improving protein interaction error rates. In CSB. IEEE, August 2004. 
[12] A. Drawid and Mark Gerstein. A Bayesian system integrating expression data with sequence patterns for localizing proteins: comprehensive application to the yeast genome. Journal of Molecular Biology, 301:1059-1075, 2000.

[13] R. Edwards and L. Glass. Combinatorial explosion in model gene networks. Chaos, 10:691-704, September 2000.

[14] R. Finn, M. Marshall, and A. Bateman. ipfam: visualization of protein-protein interactions in pdb at domain and amino acid resolutions. Bioinformatics, 21:410-412, 2005.

[15] A.-C. Gavin et al. Functional organization of the yeast proteome by systematic analysis of protein complexes. Nature, 415:141-147, 2002.

[16] A.-C. Gavin et al. Proteome survey reveals modularity of the yeast cell machinery. Nature, 440:631-636, 2006.

[17] M.A. Gilchrist, L.A. Salter, and A. Wagner. A statistical framework for combining and interpreting proteomic datasets. Bioinformatics, 20:689-700, 2004.

[18] S.M. Gomez, W.S. Noble, and A. Rzhetsky. Learning to predict protein-protein interactions. Bioinformatics, 19:1875-1881, 2003.

[19] S.M. Gomez and A. Rzhetsky. Towards the prediction of complete protein-protein interaction networks. In PSB, volume 7, pages 413-424, 2002.

[20] Y. Ho et al. Systematic identification of protein complexes in Saccharomyces cerevisiae by mass spectrometry. Nature, 415:123-124, 2002.

[21] T. Ito, T. Chiba, R. Ozawa, M. Yoshida, M. Hattori, and Y. Sakaki. A comprehensive two-hybrid analysis to explore the yeast protein interactome. PNAS, 98:4569-4574, 2001.

[22] R. Jansen, H. Yu, D. Greenbaum, Y. Kluger, Krogan N.J., Chung S., Emili A., Snyder M., Greenblatt J.F., and Gerstein M. A Bayesian networks approach for predicting proteinprotein interactions from genomic data. Science, 302:449-453, 2003.

[23] N.J. Krogan et al. High-definition macromolecular composition of yeast rna-processing complexes. Molecular Cell, 13:225-39, 2004.

[24] N.J. Krogan et al. Global landscape of protein complexes in the yeast saccharomyces cerevisiae. Nature, 440:637-643, 2006.

[25] R. Lyons and Y. Peres. Probability on trees and networks. Cambridge University Press, in progress, 2005.

[26] S. Martin, D. Roe, and J.-L. Faulon. Predicting proteinprotein interactions using signature products. Bioinformatics, 21:218-226, 2005.

[27] H.W. Mewes, D. Frishman, U. Guldener, G. Mannhaupt, K. Mayer, M. Mokrejs, B. Morgenstern, M. Munsterkotter, S. Rudd, and B. Weil. MIPS: a database for genomes and protein sequences. Nucleic Acids Research, 30:31-34, 2002.

[28] N.J. Mulder et al. Interpro, progress and status in 2005. Nucleic Acids Research, 33:D201D205, 2005.

[29] T.M. Nye, C. Berzuini, W.R. Gilks, M.M. Babu, and S.A. Teichmann. Statistical analysis of domains in interacting protein pairs. Bioinformatics, 21:993-1001, 2005.

[30] T.M. Nye, C. Berzuini, W.R. Gilks, M.M. Babu, and S.A. Teichmann. Predicting the strongest domain-domain contact in interacting protein pairs. Statistical Applications in Genetics and Molecular Biology, 5, 2006.

[31] D. Scholtens, M. Vidal, and R. Gentleman. Local modeling of global interactome networks. Bioinformatics, 21:3548-3557, 2005.

[32] E. Sprinzak and H. Margalit. Correlated sequence-signatures as markers of protein-protein interaction. J. Mol. Biol., 311:681-692, 2001.

[33] A. Stein, R. Russell, and P. Aloy. 3did: interacting protein domains of known threedimensional structure. Nucleic Acids Research, 33:D413-D417, 2005. 
[34] P. Uetz, L. Giot, G. Cagney, T.A. Mansfield, R.S. Judson, V. Narayan, D. Lockshon, M. Srinivasan, P. Pochart, A. Qureshi-Emili, Y. Li, B. Godwin, D. Conover, T. Kalbfleisch, G. Vijayadamodar, M. Yang, M. Johnston, S. Fields, and J.M. Rothberg. A comprehensive analysis of protein-protein interactions in Saccharomyces cerevisiae. Nature, 403(6770):623-627, 2000.

[35] C. von Mering, R. Krause, B. Snel, M. Cornell, S.G. Oliver, S. Fields, and P. Bork. Comparative assessment of large-scale data sets of protein-protein interactions. Nature, 417: 399-403, 2002.

[36] H. Wang, E. Segal, A. Ben-Hur, D. Koller, and D. Brutlag. Identifying protein-protein interaction sites on a genome-wide scale. In Advances in Neural Information Processing Systems (NIPS 2004), Vancouver, Canada, 2004.

[37] D.B. Wilson. Generating random spanning trees more quickly than the cover time. In Symposium on Theory of Computing, pages 396-303. ACM, 1996.

[38] I. Xenarios, L. Salwinski, X.J. Duan, P. Higney, S.M. Kim, and D. Eisenberg. DIP, the database of interacting proteins: a research tool for studying cellular networks of protein interactions. Nucleic Acids Research, 30:303-305, 2002.

[39] L.V. Zhang, S.L. Wong, O.D. King, and F.P. Roth. Predicting co-complexed protein pairs using genomic and proteomic data integration. BMC Bioinformatics, 5:1-15, 2004. 